\title{
Bone status in adolescents and young adults with type 1 diabetes: a 10-year longitudinal study
}

\author{
Agata Chobot ${ }^{(1}$, Oliwia Janota ${ }^{\circledR 2}$, Katarzyna Bąk-Drabik ${ }^{\circledR 3}$, Joanna Polanska ${ }^{(1)}$, \\ Wojciech Pluskiewicz ${ }^{105}$ \\ ${ }^{1}$ Department of Pediatrics, Institute of Medical Sciences, University of Opole, Opole, Poland \\ ${ }^{2}$ Department of Children's Diabetology, Faculty of Medical Sciences in Katowice, Medical University of Silesia, Katowice, Poland \\ ${ }^{3}$ Department of Pediatrics, Faculty of Medical Sciences in Zabrze, Medical University of Silesia, Katowice, Poland \\ ${ }^{4}$ Department of Data Sciences and Engineering, Silesian University of Technology, Gliwice, Poland \\ ${ }^{5}$ Department of Metabolic Bones Diseases, Faculty of Medical Sciences in Zabrze, Medical University of Silesia, Katowice, Poland
}

\begin{abstract}
Introduction: This study presents a 10-year longitudinal assessment of bone status in adolescents and young adults with type 1 diabetes (T1D).

Material and methods: Thirty-two patients (12 female, aged $20.5 \pm 3.93$ years, T1D duration $13.9 \pm 1.97$ years) were studied using quantitative ultrasound (QUS) and dual-energy X-ray absorptiometry (DXA). Standard deviation scores (SDS) for these results were calculated. The following clinical parameters were analysed: sex, age, T1D duration, anthropometric parameters, daily insulin requirement (DIR), mean glycated haemoglobin $\left(\mathrm{HbA}_{1 c}\right)$ in the year preceding the examination, medication other than insulin, history of bone fractures, and comorbidities.

Results: The current and past (measured 10 years earlier) QUS results did not differ and showed a significant correlation $(\mathrm{r}=0.55$, $\mathrm{p}=0.001$ ). We found no relation of QUS results and anthropometric parameters or gender. DXA parameters did not correlate with the present QUS measurement. DXA and QUS results were independent of HbA1c, co-morbidities, or intake of additional medicaments. Conclusions: Bone status parameters of the examined patients with currently suboptimal glycaemic control were found to be lowered in comparison to a normative reference population, both at baseline and follow-up, although no further deterioration was observed during the 10-year follow-up period. (Endokrynol Pol 2020; 71 (6): 532-538)
\end{abstract}

Key words: type 1 diabetes; glycaemic control; bone status; quantitative ultrasound; dual X-ray absorptiometry

\section{Introduction}

Bone mass in children and adolescents is highly dependent on environmental and hormonal factors. During this critical period of the skeleton's development, the majority of type 1 diabetes (T1D) cases are diagnosed. In the last decades, several studies concerning the influence of type 2 as well as type 1 diabetes on bone were published $[1,2]$. The pathomechanism of the development of bone changes includes factors like insulinopaenia, deficiency of growth hormone and factors (e.g. insulin-like growth factor IGF-1), hypercalciuria associated with glucosuria, improper collagen I synthesis [3], as well as advanced glycation end products (AGEs) accumulating likewise in bones [4]. It has been suggested that bone disease might be another manifestation of microvascular disease in T1D [1].

The results of some previously performed studies indicate that unsatisfactory metabolic control, long DM1 duration, low pubertal stage (especially in newly diagnosed patients), high daily insulin dose [5-7], low body mass index, reduced renal function, and the presence of diabetic complications are clinical factors of increased risk of decreased bone status or reduced bone mineral density (BMD) [8]. Nonetheless, the outcomes of studies are ambiguous, and other authors disclaim any influence of metabolic control, disease duration, and insulin requirement on $\operatorname{BMD}[9,10]$, or they suggest that the impact of diabetes-specific parameters on bone mineral density is low [11, 12]. Interestingly, bone status assessment in subjects having T1D for 50 or more years showed normal results, and the fracture risk among patients was comparable to non-diabetic peers [13].

The gold standard for diagnosing changes in bone is two-dimensional X-ray absorptiometry (DXA). Although it is a widely used technique and is best for precise assessment, its application for screening purposes in the paediatric population is a point of argument, mainly due to the exposure to X-ray radiation $[14,15]$. 
Quantitative ultrasound (QUS) is a simple, non-invasive, and cost-effective method. More importantly, it does not require the use of radiation. These advantages could favour its use for bone status investigation in paediatrics, but there are some controversies because it does not assess bone structure and geometry separately (parameters influenced by age, height, and timing of sexual maturation). Nonetheless, studies have confirmed the utility of phalangeal QUS in the assessment of bone status in children, adolescents, and young adults, with a very small confounding effect related to bone size, as well as in monitoring longitudinal changes [14-17].

In 2007 in the region of Silesia, Poland, two studies of bone quality measured by QUS amongst children and adolescents with T1D were performed [3,6]. Bone quality of adolescents with T1D was significantly worse than that of healthy peers. Moreover, those with $\mathrm{HbA}_{1 \mathrm{c}}>7.0 \%$ (> $53 \mathrm{mmol} / \mathrm{mol}$ ) had a significantly lower amplitude-dependent speed of sound (Ad-SoS SDS) [3]. T1D duration in pre-pubertal children, as well as adolescents, was negatively related to QUS measurement outcomes [3, 6]. Gender, long- and short-term metabolic control, and history of acute diabetes complications had no impact on bone quality.

This study aimed to reassess the bone status of patients with T1D and compare results with those were obtained 10 years earlier, as well as to estimate the impact of diabetes-related parameters on bone

\section{Material and methods}

\section{Patients}

During the initial study -10 years earlier -182 children and adolescents with T1D were examined [6]. Based on the last known patients' contact details from the database of the outpatient clinic as well as hospital record from the Department of Children's Diabetology in Katowice, Poland, no less than three attempts at telephone contact were made, to invite patients for participation in the current study. Additionally, information about the investigation was given to diabetology clinics on the territory of Upper Silesia. However, many of the contact details from the paediatric diabetes centre were out of date, and some of the patients had moved to other cities or abroad and could not take part in the examination even if various dates for appointments were offered. Ultimately 32 patients (20 men, 12 women) aged 12.8-27.62 (20.5 \pm 3.93$)$ years with DM1 lasting 11.3-17.9 (13.9 \pm 1.97$)$ years agreed to take part and appeared for the examinations. The general characteristics of the study group are presented in Table 1 .

Table 1. Characteristics of the study group. Values given as total number (n) and percentage or mean \pm standard deviation and $95 \%$ confidence interval

\begin{tabular}{|c|c|c|c|c|}
\hline \multirow{2}{*}{ Gender } & All patients & Females & Males & \multirow{2}{*}{$\begin{array}{c}p \text { value } \\
\text { and effect size }\end{array}$} \\
\hline & $32(100 \%)$ & $12(37.5 \%)$ & $20(62.5 \%)$ & \\
\hline \multirow{2}{*}{ Age [yrs] } & $20.5 \pm 3.9$ & $21.4 \pm 3.8$ & $19.9 \pm 4.0$ & 0.30 (ANOVA) \\
\hline & $(19.1,21.9)$ & $(-0.48,0.78)$ & $(-0.56,0.53)$ & d0.38 - small \\
\hline \multirow{2}{*}{ Type 1 diabetes duration [yrs] } & $13.88 \pm 1.97$ & $14.42 \pm 2.28$ & $13.55 \pm 1.73$ & 0.23 (ANOVA) \\
\hline & $(1.2,14.6)$ & $(12.99,15.85)$ & $(12.74,14,36)$ & d0.44 - small \\
\hline \multirow{2}{*}{ Weight SDS } & $0.53 \pm 0.90$ & $0.99 \pm 0.71$ & $0.25 \pm 0.90$ & 0.02 (ANOVA) \\
\hline & $(0.20,0.85)$ & $(0.54,1.44)$ & $(-0.17,0.67)$ & d0.88 - large \\
\hline \multirow{2}{*}{ Height SDS } & $0.05 \pm 1.10$ & $0.15 \pm 1.00$ & $-0.02 \pm 1.17$ & 0.68 (ANOVA) \\
\hline & $(-0.35,0.44)$ & $(-0.48,0.78)$ & $(-0.56,0.53)$ & d0.15 - none \\
\hline \multirow{2}{*}{ Body mass index SDS } & $0.58 \pm 0.85$ & $1.05 \pm 0.75$ & $0.29 \pm 0.79$ & 0.01 (ANOVA) \\
\hline & $(0.27,0.88)$ & $(0.58,1.52)$ & $(-0.07,0.66)$ & d0.98 — large \\
\hline \multirow{2}{*}{$\mathrm{HbA}_{1 \mathrm{c}}$ most recent (\%) } & $8.04 \pm 1.64$ & $7.38 \pm 0.85$ & $7.91 \pm 1.48$ & 0.29 (ANOVA) \\
\hline & $(7.44,8.64)$ & $(7.05,9.47)$ & $(7.19,8.61)$ & d0.21 - small \\
\hline \multirow{2}{*}{$\mathrm{HbA}_{1 \mathrm{c}}$ mean last year (\%) } & $8.13 \pm 1.49$ & $8.01 \pm 1.24$ & $8.02 \pm 1.30$ & 0.99 (ANOVA) \\
\hline & $(7.58,8.68)$ & $(7.25,9.48)$ & $(7.36,8.61)$ & d0.25 - small \\
\hline \multirow{2}{*}{ DIR [U/24 h] } & $0.78 \pm 0.21$ & $0.77 \pm 0.19$ & $0.78 \pm 0.22$ & 0.92 (ANOVA) \\
\hline & $(0.70,0.85)$ & $(0.65,0.89)$ & $(0.67,0.89)$ & d0.04 - none \\
\hline Additional diseases* $\left.{ }^{*} \mathrm{Yes} / \mathrm{No}\right]$ & $10(31.3 \%) / 22(68.7 \%)$ & $6(50 \%) / 6(50 \%)$ & $4(20 \%) / 16(80 \%)$ & $0.08\left(\chi^{2}\right)$ \\
\hline Fractures [Yes/No] & $\begin{array}{c}10(31.25 \%) / 22 \\
(68.75 \%)\end{array}$ & $12(100 \%) / 0(0 \%)$ & $10(50 \%) / 10(50 \%)$ & $<0.01\left(\chi^{2}\right)$ \\
\hline Additional medication [Yes/No] & $\begin{array}{c}10(31.25 \%) / 22 \\
(68.75 \%)\end{array}$ & $5(41.67 \%) / 7(58.33 \%)$ & $5(25 \%) / 15(75 \%)$ & $0.32\left(\chi^{2}\right)$ \\
\hline
\end{tabular}

SDS - standard deviation score; $\mathrm{HbA}_{1 \mathrm{c}}$ — haemoglobin $\mathrm{A}_{1 \mathrm{c}}$; $\mathrm{DIR}$ — daily insulin requirement; ${ }^{*}$ presence of any additional disease or medication that is known to influence bone health 


\section{Methods}

Clinical data of all of the 32 patients were collected. In the whole group, bone status was examined by QUS (using the same device as 10 years earlier), and 24 patients also consented for DXA measurements

\section{Clinical data}

Data were collected in a compendious, standardised questionnaire. They included the history of any bone fractures, medications, and comorbidities (those that are known to impact bone health or metabolism were considered as relevant). Patients were also asked to give detailed information concerning diabetes: date of diagnosis, present daily insulin dose, and the number of severe acute diabetes complications in the past year [diabetic ketoacidosis (DKA) and severe hypoglycaemia $(\mathrm{SH})$ episodes]. Given the daily insulin dose and weight, the daily insulin requirement (DIR) was calculated for each patient. Because the group consisted of adolescents and young adults, height, weight, and the calculated body mass index (BMI) were expressed using the standard deviation score (SDS).

\section{Bone measurements}

Quantitative ultrasound measurements [DBM Sonic 1200 device (IGEA, Carpi, Italy)] were performed at the distal end of the proximal phalanges II to $\mathrm{V}$ of both hands. A mean of the amplitudedependent speed of sound (Ad-SoS) values obtained for all fingers of the non-dominant hand was calculated. In order to normalise the result for age and gender, an SDS was calculated using a standard Z-score transformation with mean value and standard deviation appropriate for age and sex group. Normative data for phalangeal QUS have been published (17). QUS assessment was done by one experienced investigator. For the performed quantitative ultrasound measurements, the calculated coefficient of variation $(\mathrm{CV} \%)$ equalled $0.64 \%$.

Bone densitometry (DXA measurements) was carried out through Hologic Explorer (Hologic Inc., Waltham, MA, USA; software version: 13.0:3). Bone mineral density (BMD $\left[\mathrm{g} / \mathrm{cm}^{2}\right]$ ) for total body (TB-BMD) and lumbar spine (s-BMD) was estimated. For the obtained values, respective SDS values were calculated using gender- and age-specific reference data from Hologic Explorer. All measurements were performed by the same experienced technician - the intra-individual precision $(\mathrm{CV} \%)$ of the measurements was $1.8 \%$ for TB-BMD and $1.6 \%$ for s-BMD.

As described above for the QUS and DXA results, the SDS was calculated. The Z-score transformation is necessary to achieve a normalisation that allows comparison between children of different ages and genders. The narrow normal range of SDS is defined as -1.0 to 1.0 , and a wide range from -2.0 to 2.0. Based on DXA, if there are no clinically significant fractures, "low BMD" should be diagnosed when BMD SDS $\leq-2.0$ [18].

The study was approved by the Ethics Committee of the Medical University of Silesia, Katowice, Poland (SUM NN-6501-95/06), and written informed consent was received from participants prior to inclusion in the study.

The database can be provided on request.

\section{Statistical analysis}

Statistical analyses were performed using R software (http://www. bioconductor.org/). Descriptive statistics - number (n), median, upper and lower quartile (and), minimal (min) and maximal (max) value, mean, and standard deviation (SD) - were determined for all of the analysed parameters. We used Tukey's test to identify outlying values and the Lilliefors parametric test to assess the normality of the distribution. The variance homogeneity hypothesis was tested using a Bartlett statistic. Comparative analysis of normally distributed variables was carried out by analysis of variance (ANOVA) or Student's t-test depending on the group number. In cases of other types of distribution, the nonparametric ANOVA Kruskal-Wallis and Mann-Whitney test were employed.
Correlations between two continuous variables were assessed using Pearson's or Spearman's correlation coefficients. We applied the Wilcoxon signed rank test to assess changes in time. Statistical significance was considered at $\mathrm{p}<0.05$.

\section{Results}

\section{General characteristics of the study group}

Table 1 presents the characteristics of the study group. The most recent $\mathrm{HbA}_{1 \mathrm{c}}$ results were $6.0-12.7 \%$ $(8.04 \pm 1.64 \%)(42-115 \mathrm{mmol} / \mathrm{mol})$, and mean glycated haemoglobin from the last year was $6.28-12.63 \%$ $(8.13 \pm 1.49 \%)(45-116 \mathrm{mmol} / \mathrm{mol})$, which allows us to consider the patients' metabolic control as insufficient. The most recent $\mathrm{HbA}_{1 \mathrm{c}}$ value and the last year's mean had strong, positive correlation $(r=0.86$, $\mathrm{p}<0.001)$.

The mean daily insulin requirement was $0.78 \pm 0.21$ unit $/ \mathrm{kg}$. Twenty-two patients declared to have other comorbidities and intake of additional medication. Also, 22 patients had bone fractures (in most cases forearm or wrist, two clavicula fractures, one tibia fracture, one metatarsal bone fracture, and one of the orbital bones). All fractures reported by the patients were related to significant trauma. There were some gender differences among the study group. Weight SDS values $(0.99 \pm 0.71$ vs. $0.25 \pm 0.90, p=0.022)$ were higher in females but BMI SDS values $(1.05 \pm 0.75$ vs. $0.29 \pm 0.79, \mathrm{p}=0.011)$ were higher in males. Moreover, the occurrence of fractures was higher among girls than boys (100\% vs. $50 \%$, $\mathrm{p}=0.003)$. Other parameters did not differ between genders or subgroups with and without positive bone fracture history.

Age was positively related to weight SDS $(r=0.41$, $\mathrm{p}=0.019)$ as well as to BMI SDS $(\mathrm{r}=0.48, \mathrm{p}=0.006)$. Also, the duration of DM1 was positively associated with weight SDS $(\mathrm{r}=0.48, \mathrm{p}=0.006)$ and BMI SDS $(\mathrm{r}=0.49, \mathrm{p}=0.005)$. Weight SDS was associated with height SDS ( $\mathrm{r}=0.54, \mathrm{p}=0.001)$ and BMI SDS ( $\mathrm{r}=0.81$, $\mathrm{p}<0.001)$. Surprisingly, we observed also a negative correlation of weight SDS as well as BMI SDS and daily insulin requirement $(\mathrm{r}=-0.52, \mathrm{p}=0.003$ and $\mathrm{r}=-0.42$, $\mathrm{p}=0.022$, respectively).

\section{QUS measurements}

Mean Ad-SoS SDS for the whole study group was $-0.22 \pm 1.25$ (95\% CI: $-0.67,0.23)$. Analysis using paired tests showed no significant change (mean $\delta$ Ad-SoS SDS $-0.13 \pm 1.09$ [95\% CI: $-0.53,0.26])$ in Ad-SoS SDS between the current and past assessment $(-0.09 \pm 1.03$ [95\% CI: $-0.46,0.28]$ ) (Tab. 2). We revealed a significant correlation of the past and present Ad-SoS SDS results $(\mathrm{r}=0.55, \mathrm{p}=0.001)$ (Fig. 1), as well as a positive association between the current QUS measurement result and its change in time $-\delta$ Ad-SoS SDS $(r=0.62, p<0.0001)$. 
Table 2. Quantitative ultrasound measurement results during the current study, baseline measured 10 years earlier, and the difference - data presented as mean, standard deviation and 95\% confidence interval

\begin{tabular}{lcccc}
\hline & All patients & Females (F) & Males (M) & F vs. M \\
\hline Ad-SoS SDS baseline & $-0.09 \pm 1.03$ & $-0.06 \pm 1.04$ & $-0.10 \pm 1.05$ & $\mathrm{p}=0.276$ \\
(10 years earlier) & $(-0.46,0.28)$ & $(-0,72,0.59)$ & $(-0.59,0.39)$ & Small effect size \\
\hline \multirow{2}{*}{ Ad-SoS SDS current } & $-0.22 \pm 1.25$ & $0.71 \pm 1.32$ & $0.07 \pm 1.42$ & $\mathrm{p}=0.088$ \\
& $(-0.67,0.23)$ & $(-1.54,0.12)$ & $(-0.46,0.60)$ & Medium effect size \\
\hline \multirow{2}{*}{$\delta$ Ad-SoS SDS } & $-0.13 \pm 1.09$ & $0.17 \pm 1.00$ & $-0.65 \pm 1.09$ & $\mathrm{p}=0.038$ \\
& $(-0.53,0.26)$ & $(-0.29,0.64)$ & $(-1.33,0.04)$ & Medium effect size \\
\hline
\end{tabular}

Ad-SoS SDS — amplitude-dependent speed of sound standard deviation score; $\delta$ Ad-SoS SDS — difference in Ad-SoS SDS between the current and baseline measurement

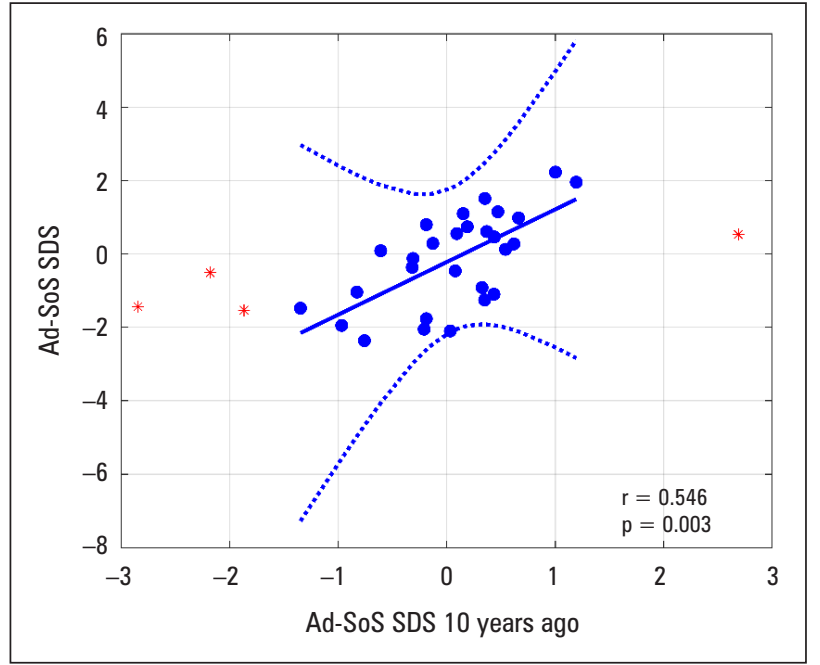

Figure 1. Correlation of present amplitude-dependent speed of sound standard deviation score (Ad-SoS SDS) and the quantitative ultrasound results from 10 years earlier $(r=0.55, p=0.001)$

There were no gender differences in the present Ad-SoS SDS [female vs. male: $-0.71 \pm 1.32$ (95\% CI: $-1.54,0.12)$ vs. $0.07 \pm 1.42$ (95\% CI: $-0.46,0.60)$, Cohen's $\mathrm{d}=0.64-$ medium effect size] as well as in the QUS measurement results from 10 years earlier (Tab. 2, rank biserial correlation coefficient 0.20 - small effect size). Nevertheless, the change in time in Ad-SoS SDS $(\delta$ Ad-SoSSDS - the difference between the present value and the one measured 10 years earlier) was greater in males [0.17 \pm 1.00 (95\% CI: $-0.29,0.64)$ vs. $-0.65 \pm 1.09$ (95\% CI: $-1.33,0.04), p=0.038$, Cohen's d $=0.79-$ medium effect size]. We found no relation between Ad-SoS SDS and anthropometric parameters.

\section{DXA measurements and their relation to present QUS results}

The mean TB-BMD SDS in the study group was $-1.53 \pm 0.75$ (95\% CI: $-1.87,-1.19)$ and s-BMD SDS $-0.39 \pm 0.98$ (95\% CI: $-0,80,0,02)$.

TB-BMD SDS was positively associated with age $(\mathrm{r}=0.54, \mathrm{p}=0.012)$, weight SDS $(\mathrm{r}=0.62, \mathrm{p}=0.003)$, height SDS ( $r=0.46, p=0.036)$, and BMI SDS ( $r=0.47$, $\mathrm{p}=0.032)$, but we found no correlation between the SDS of the anthropometric parameters and s-BMD SDS.

TB-BMD SDS was significantly, positively related to $s-B M D$ SDS ( $r=0.61, p=0.003)$. TB-BMD SDS increased along with $\delta$ Ad-SoS SDS $(\mathrm{r}=0.57, \mathrm{p}=0.021)$, but we could not confirm any association between DXA parameters and the present Ad-SoS SDS.

\section{Bone measurements and diabetes-related parameters}

Glycosylated haemoglobin $\left(\mathrm{HbA}_{1 \mathrm{c}}\right)$ values - most recent and the mean from the last year - were not associated with Ad-SoS SDS or $\delta$ Ad-SoS SDS. Also, no relation of $\mathrm{HbA}_{1 \mathrm{c}}$ values with either TB-BMD SDS or s-BMD SDS was found. We also found no associations of bone measurement results, regardless of the method applied, and either disease duration or DIR. Detailed data are presented in Table 3.

Bone measurements, fractures, comorbidities, and additional medication

All fractures reported by the patients were non-vertebral and high-energy fractures. Presence of comorbidities or history of additional medication did not impact the results of the QUS measurement. Also, all parameters assessed using DXA were not influenced by comorbidities and additional medication.

\section{Discussion}

We showed that in our patients, bone status measured using QUS did not change during a decade. The past and present results were in positive correlation, as was the current Ad-SoS SDS and its change in time $(\delta$ Ad-SoS SDS). Both means of the DXA results - total body and spine - were significantly decreased. Although the decrease is smaller than 2 SDS, it can be considered as clinically relevant.

In our study, QUS outcomes were independent of the results of bone assessment using the gold standard 
Table 3. Correlations between current results of bone measurements and diabetes-related parameters

\begin{tabular}{|c|c|c|c|c|}
\hline & Ad-SoS SDS & $\delta$ Ad-SoS SDS & TB-BMD SDS & s-BMD SDS \\
\hline $\mathrm{HbA}_{1 \mathrm{c}}$ mean from last year & $r=-0.17, p=0.354^{s}$ & $r=-0.15, p=0.438^{s}$ & $r=0.08, p=0.711^{n}$ & $r=0.04, p=0.846^{n}$ \\
\hline $\mathrm{HbA}_{1 \mathrm{c}}$ most recent & $r=-0.17, p=0.372^{s}$ & $r=-0.14, p=0.449^{s}$ & $r=-0.01, p=0.977^{n}$ & $r=0.12, p=0.594^{s}$ \\
\hline T1D duration & $r=-0.21, p=0.257^{s}$ & $r=0.02, p=0.908^{n}$ & $r=0.33, p=0.151^{m}$ & $r=-0.04, p=0.867^{n}$ \\
\hline DIR & $r=0.01, p=0.941^{n}$ & $r=-0.23, p=0.219^{s}$ & $r=-0.39, p=0.099^{m}$ & $r=-0.18, p=0.416^{s}$ \\
\hline
\end{tabular}

Correlation coefficient and its $\mathrm{p}$ value, the effect size is marked, respectively: ${ }^{\mathrm{n}}$ — none, ${ }^{\mathrm{s}}$ — small, $\mathrm{m}$-medium; Ad-SoS SDS — amplitude-dependent speed of sound standard deviation score; $\delta$ Ad-SoS SDS — difference in Ad-SoS SDS between the current and baseline measurement; HbA diabetes; DIR — daily insulin requirement; TB-BMD SDS — total body bone mineral density standard deviation score; s-BMD SDS — spine bone mineral density standard deviation score

DXA (TB-BMD SDS and s-BMD SDS). However, interestingly, TB-BMD SDS was positively associated with the change in time in Ad-SoS SDS ( $\delta$ Ad-SoS SDS). Diabetes-related parameters such as glycaemic control, DIR, and disease duration did not impact bone status, regardless of the method applied. Although among our patients fractures were more common in females, history of fractures, comorbidities, and additional medication did not influence the bone measurement results.

The results of studies concerning the relation of T1D and bone status are ambiguous. The specific pathophysiology of bone disorders in diabetes and the correlation between these medical conditions is still an open question. One of the most recent publications regarding bone diseases in individuals with T1D suggests that it is one of the manifestations of microvascular complications [1].

Most studies indicate a negative influence of T1D on bone tissue, also in adolescents and young adults [ 1 , 19-21]. In our research the bone status of the studied patients had not deteriorated 10 years after the initial examination, although the QUS results obtained at baseline in adolescents were lower than those in healthy controls $(-0.34$ [95\% CI: $-0.57,-0.11]$ vs. $-0.03[95 \%$ CI: $-0.15,0.08], \mathrm{p}<0.05)$ [6]. Although there was a statistically significant difference, the results were within normal ranges, and the results did not deteriorate with time. Other authors $[8,9]$ disclaim the influence of diabetes-specific parameters on BMD or affirm that their impact is low [11-13, 22]. Vazquez et al. showed that children and adolescents with non-complicated T1D have no alteration of bone mass, but the physiological increase of bone density is smaller than in healthy peers. We found no relation between diabetes-related parameters and bone status assessed by either of the two methods - QUS and DXA. Our previous study, contrary to the current observation, showed a significant association between $\mathrm{HbA1c}$ and the QUS results $(\mathrm{r}=-0.22, \mathrm{p}<0.05)$ [6]. For both correlations the coefficients were quite similar, with a small effect size, so this difference may partially be explained by the diverse number of study participants. Moreover, the baseline study was carried out during the most dynamic period in bone changes, whereas the current measurements concerned mostly people in whom skeletal maturation should be completed - in this case the impact of glycaemic fluctuations might be less pronounced.

We should emphasise that our present and previous studies examined young people. Publications that describe the results of bone measurements in the population of adults with long-term type 1 diabetes $[23,24]$ did not confirm any significant differences in BMD compared to healthy controls. Maddaloni et al. examined a population of aging patients with T1D, which lasted 50 years or more (mean $\mathrm{HbA}_{1 \mathrm{c}} 7.1 \%$ ). Interestingly, their investigation exhibited low rates of non-vertebral fractures, normal results of the DXA examination, and a comparable risk of fracture in T1D patients compared to non-diabetic peers [13]. Their findings, as well as those of some other studies and our research, indicate that T1D duration is not a significant factor influencing bone status [22, 25].

We measured diabetics' bone density by quantitative ultrasound (QUS) and two-dimensional X-ray absorptiometry (DXA) methods. QUS measurements were used in the previous research (10 years ago) because the study group consisted of children and youths [6]. Outcomes of the ultrasound method are less dependent on bone size, patients are not exposed to radiation [26], and its results are comparable to those of DXA [14]. In this follow-up study, we applied both methods [3]. Despite the fact that the obtained QUS and DXA results showed, similarly, no significant deterioration of skeletal status, we could not confirm any correlation between the outcomes. This suggests that, regardless of some propositions from other studies [27], these methods cannot be used alternatively to assess bone status in T1D patients.Our analysis indicates that past and present Ad-SoS measurements were significantly correlated, as were the present result and $\delta$ Ad-SoS SDS. There were no differences between genders, although $\delta$ Ad-SoS SDS was greater in males, and fractures were more common in females. Diabetes-related parameters did not affect the bone status or its changes. Salvatoni 
et al. in their 2-year follow-up study likewise concluded that neither metabolic control nor microvascular complications impact the bone status.

We observed increasing weight and BMI SDS with age and T1D duration. This is in concordance with results obtained by other authors [28]. It is worth underlining that this is one of the few follow-up studies that compare bone status between adolescence and young adulthood in the same group of patients. The main limitation of this study is the sample size, the reasons for which we described above. There were also no DXA measurements performed during the initial assessment, and therefore the longitudinal analysis concerns only bone status assessed using QUS.

\section{Conclusions}

The bone status parameters of the examined patients with currently suboptimal glycaemic control were found to be lowered in comparison to a normative reference population, both at baseline (measured using QUS) and follow-up (QUS and DXA measurements), although no further deterioration was observed during the 10-year follow-up period. Glycaemic control was currently suboptimal in the studied group and did not influence bone status examined by either of the methods. Therefore, it seems, that potential changes in bone in T1D develop very slowly and are not directly related to glycaemic control.

\section{Acknowledgements}

We thank the patients for their participation. The authors also thank KP for his help in language editing. This work was co-financed by an SUT grant for maintaining and developing research potential.

\section{Funding}

This work was co-financed by an SUT grant for maintaining and developing research potential.

\section{References}

1. Shanbhogue VV, Hansen S, Frost M, et al. Bone disease in diabetes: another manifestation of microvascular disease? Lancet Diabetes Endocrinol. 2017; 5(10): 827-838, doi: 10.1016/S2213-8587(17)30134-1, indexed in Pubmed: 28546096.

2. Miazgowski T, Krzyzanowska-Swiniarska B, Ogonowski J, et al. [Does type 2 diabetes predispose to osteoporotic bone fractures?]. Endokrynol Pol. 2008; 59(3): 224-229, indexed in Pubmed: 18615397.

3. Chobot AP, Haffke A, Polanska J, et al. Ouantitative ultrasound bone measurements in pre-pubertal children with type 1 diabetes. Ultrasound Med Biol. 2012; 38(7): 1109-1115, doi: 10.1016/j.ultrasmedbio.2012.02.012, indexed in Pubmed: 22542259.

4. Oei L, Rivadeneira F, Zillikens MC, et al. Diabetes, diabetic complications, and fracture risk. Curr Osteoporos Rep. 2015; 13(2): 106-115 doi: 10.1007/s11914-015-0260-5, indexed in Pubmed: 25648962

5. Camurdan MO, Ciaz P, Bideci A, et al. Role of hemoglobin A(1c), duration and puberty on bone mineral density in diabetic children. Pediat Int. 2007; 49(5): 645-651, doi: 10.1111/j.1442-200X.2007.02451.x, indexed in Pubmed: 17875093.
6. Chobot AP, Haffke A, Polanska I, et al. Bone status in adolescents with type 1 diabetes. Diabetologia. 2010; 53(8): 1754-1760, doi: 10.1007/s00125-010-1782-0, indexed in Pubmed: 20454951.

7. Tsentidis C, Gourgiotis D, Kossiva L, et al. Sclerostin distribution in children and adolescents with type 1 diabetes mellitus and correlation with bone metabolism and bone mineral density. Pediatr Diabetes. 2016; 17(4): 289-299, doi: 10.1111/pedi.12288, indexed in Pubmed: 26094958.

8. Zhukouskaya VV, Eller-Vainicher C, Shepelkevich AP, et al. Bone health in type 1 diabetes: focus on evaluation and treatment in clinical practice. J Endocrinol Invest. 2015; 38(9): 941-950, doi: 10.1007/s40618-015-0284-9, indexed in Pubmed: 25863666.

9. Bechtold S, Dirlenbach I, Raile K, et al. Early manifestation of type 1 diabetes in children is a risk factor for changed bone geometry: data using peripheral quantitative computed tomography. Pediatrics. 2006; 118(3): e627-e634, doi: 10.1542/peds.2005-2193, indexed in Pubmed: 16908617.

10. Hadjidakis DJ, Raptis AE, Sfakianakis M, et al. Bone mineral density of both genders in Type 1 diabetes according to bone composition J Diabetes Complications. 2006; 20(5): 302-307, doi: 10.1016/j.jdiacomp.2005.07.006, indexed in Pubmed: 16949517

11. Catalano A, Morabito N, Di Vieste G, et al. Phalangeal quantitative ultrasound and metabolic control in pre-menopausal women with type 1 diabetes mellitus. J Endocrinol Invest. 2013; 36(5): 347-251, doi: 10.3275/8646, indexed in Pubmed: 23027769.

12. Roggen I, Gies I, Vanbesien J, et al. Trabecular bone mineral density and bone geometry of the distal radius at completion of pubertal growth in childhood type 1 diabetes. Horm Res Paediatr. 2013; 79(2): 68-74, doi: 10.1159/000346686, indexed in Pubmed: 23391966.

13. Maddaloni E, $D^{\prime}$ Eon $S$, Hastings $S$, et al. Bone health in subjects with type 1 diabetes for more than 50 years. Acta Diabetol. 2017; 54(5): 479-488, doi: 10.1007/s00592-017-0973-2, indexed in Pubmed: 28236093.

14. Baroncelli GI. Quantitative ultrasound methods to assess bone mineral status in children: technical characteristics, performance, and clinical application. Pediatr Res. 2008; 63(3): 220-228, doi: 10.1203/PDR.0b013e318163a286, indexed in Pubmed: 18287958.

15. Halupczok-Żyła J, Gojny Ł, Bolanowski M. Trabecular bone score (TBS) as a noninvasive and complementary tool for clinical diagnosis of bone structure in endocrine disorders. Endokrynol Pol. 2019; 70(4): 350-356, doi: 10.5603/EPa2019.0022, indexed in Pubmed: 31489959.

16. Baroncelli GI, Federico G, Vignolo M, et al. Phalangeal Quantitative Ultrasound Group. Cross-sectional reference data for phalangeal quantitative ultrasound from early childhood to young-adulthood according to gender, age, skeletal growth, and pubertal development. Bone. 2006; 39(1): 159-173, doi: 10.1016/j.bone.2005.12.010, indexed in Pubmed: 16473568

17. Halaba ZP. Quantitative ultrasound measurements at hand phalanges in children and adolescents: a longitudinal study. Ultrasound Med Biol. 2008; 34(10): 1547-1553, doi: 10.1016/j.ultrasmedbio.2008.03.007, indexed in Pubmed: 18485570

18. Gordon CM, Leonard MB, Zemel BS, et al. International Society for Clinical Densitometry. 2013 Pediatric Position Development Conference: executive summary and reflections. J Clin Densitom. 2014; 17(2): 219-224, doi: 10.1016/j.jocd.2014.01.007, indexed in Pubmed: 24657108

19. Heap J, Murray MA, Miller SC, et al. Alterations in bone characteristics associated with glycemic control in adolescents with type 1 diabetes mellitus. J Pediatr. 2004; 144(1): 56-62, doi: 10.1016/j.jpeds.2003.10.066, indexed in Pubmed: 14722519.

20. Saha MT, Sievänen H, Salo MK, et al. Bone mass and structure in adolescents with type 1 diabetes compared to healthy peers. Osteoporos Int. 2009; 20(8): 1401-1406, doi: 10.1007/s00198-008-0810-0, indexed in Pubmed: 19083073

21. Fuusager GB, Christesen HT, Milandt N, et al. Glycemic control and bone mineral density in children and adolescents with type 1 diabetes. Pediatr Diabetes. 2019; 20(5): 629-636, doi: 10.1111/pedi.12861, indexed in Pubmed: 31017353.

22. Hamilton EJ, Drinkwater JJ, Chubb SA, et al. A 10-Year Prospective Study of Bone Mineral Density and Bone Turnover in Males and Females With Type 1 Diabetes. J Clin Endocrinol Metab. 2018; 103(9): 3531-3539, doi: 10.1210/jc.2018-00850, indexed in Pubmed: 30032248.

23. Ingberg CM, Palmér M, Aman J, et al. Body composition and bone mineral density in long-standing type 1 diabetes. J Intern Med. 2004; 255(3): 392-398, doi: 10.1046/j.1365-2796.2003.01283.x, indexed in Pubmed: 14871464.

24. Rakic V, Davis WA, Chubb SAP, et al. Bone mineral density and its determinants in diabetes: the Fremantle Diabetes Study. Diabetologia. 2006; 49(5): 863-871, doi: 10.1007/s00125-006-0154-2, indexed in Pubmed: 16518589 .

25. Alhuzaim ON, Lewis EJH, Lovblom LE, et al. Bone mineral density in patients with longstanding type 1 diabetes: Results from the $\mathrm{Ca}$ nadian Study of Longevity in Type 1 Diabetes. J Diabetes Complications. 2019; 33(11): 107324, doi: 10.1016/j.jdiacomp.2018.12.009, indexed in Pubmed: 31003922 
26. Halaba ZP, Konstantynowicz J, Pluskiewicz W, et al Comparison of phalangeal ultrasound and dual energy X-ray absorptiometry in healthy male and female adolescents. Ultrasound Med Biol. 2005; 31(12): 1617-1622, doi: 10.1016/j.ultrasmedbio.2005.07.013, indexed in Pubmed: 16344124.

27. Baroncelli GI, Battini R, Bertelloni S, et al. Analysis of quantitative ultrasound graphic trace and derived variables assessed at proximal phalanges of the hand in healthy subjects and in patients with cerebral palsy or juvenile idiopathic arthritis. A pilot study. Bone. 2010; 46(1): 182-189, doi: 10.1016/j.bone.2009.09.010, indexed in Pubmed: 19772958

28. Sandhu N, Witmans MB, Lemay JF, et al. Prevalence of overweight and obesity in children and adolescents with type 1 diabetes mellitus. J Pediatr Endocrinol Metab. 2008; 21(7): 631-640, doi: 10.1515/JPEM.2008.21.7.631, indexed in Pubmed: 18780597. 